\title{
Enthierarchisierung der Personalverwaltung: Ein großes Reformpotential für die deutsche Justiz
}

\section{Allgemeine Vorbemerkung \\ 1. Entfremdung zwischen Justiz und Politik}

In weiten Bereichen ist eine Entfremdung zwischen der Justiz und der Politik feststellbar. Sie resultiert unter anderem aus den in der täglichen Praxis spürbaren Mängeln in der personellen und finanziellen Ausstattung der Justiz, einer nicht immer gewährleisteten Partizipation, aber auch gelegentlichem ministeriellem »Durchregieren « hinein in das sog. operative Geschäft der Gerichte. In der aktuellen Diskussion um diese Probleme liegt eine Chance zur Veränderung.

\section{Bestimmung der Trennlinie zwischen »Selbstverwaltung « und $»$ Fremdverwaltung «}

Die gefühlte Trennlinie zwischen »Selbstverwaltung « und »Fremdverwaltung « verläuft aus Sicht der Richterschaft aber nicht nur zwischen Justizpraxis und Justizministerium. Sie wird auch gesehen im Verhältnis von Richterschaft zur jeweiligen Gerichtsverwaltung. Dieses Dilemma ist nicht lösbar durch den Austausch von Institutionen, sondern nur durch mehr Partizipation.

3. Forderung eines neuen Verständnisses der Wahrnehmung von Verwaltungsaufgaben

Eine Reform der Justiz erfordert keine neuen Institutionen (z.B. »Justizrat«). Strukturell würde sich kaum etwas ändern. ${ }^{1}$ Die Justiz von morgen braucht vielmehr ein neues Verständnis der Wahrnehmung von Verwaltungsaufgaben. Dieses sollte gekennzeichnet sein durch eine Stärkung der Kompetenzen vor Ort (Enthierarchisierung durch mehr Delegation, Kooperation und Kommunikation) und eine Konzentration der Ministerien auf strategische Steuerungsaufgaben.

\section{Größere Akzeptanz durch mehr Selbstverwaltung in Personalangelegenheiten}

Ein solcher Paradigmenwechsel findet seine besondere Bedeutung in der Personalverwaltung. Je mehr die Gerichte für die Personalangelegenheiten der Richter allein zuständig oder im Wege verstärkter Partizipation an Ministerialentscheidungen beteiligt sind, desto größer ist die Gewähr einer Akzeptanz in der Kollegenschaft.

1 Siehe ergänzend auch Isermann, Selbstverwaltung der Justiz - aber ohne neue Institutionen, Recht und Politik 2008, S. 30-34 m.w.N. 


\section{Spannungsfeld zwischen Selbstverwaltung und Qualitätssicherung}

Weil Maßnahmen der Personalauswahl und der Personalentwicklung gute Personalkenntnisse ebenso erfordern wie eine hohe Professionalität, ist eine Stärkung der Kompetenzen vor Ort von einem Spannungsverhältnis gekennzeichnet, das einer wohlbedachten Abwägung bedarf (Kenntnisse der Personen und Verhältnisse vor Ort, Stärkung der Partizipation versus Sicherung landesweit gültiger Qualitätsstandards und einheitlicher Verfahrensregeln, Meidung kooptativer Elemente).

\section{Vorbild einiger Landesjustizverwaltungen}

Einige Landesjustizverwaltungen haben bereits den Weg einer weitgehenden Delegation in Personalangelegenheiten beschritten. Die dort gemachten Erfahrungen rechtfertigen es, darin ein pragmatisch realisierbares, aber auch ausbaufähiges Modell zu sehen.

\section{II. Übersicht zur Personalverwaltungspraxis in den Bundesländern}

Ebenso wenig wie es »das« Modell der Selbstverwaltung gibt, gibt es »die deutsche Justiz«, wenn es um die Frage der Richterauswahl und Richterbeförderung geht. Der nachfolgende Überblick zur Lage in den Landesjustizverwaltungen ist vom Oberlandesgericht Hamm für die Konferenz der OLG-Präsidenten im Jahr 2005 in Braunschweig erstellt worden und zeigt, welche Kompetenzen den Oberlandesgerichten als der oberen Justizbehörde unterhalb der politischen Ebene der Ministerien (oberste Justizbehörde) zukommen. Er orientiert sich an den Stadien einer beruflichen Entwicklung, also an dem Auswahlverfahren bei der Einstellung von Richtern (Ersternennung), der Anstellung von Richtern (Ernennung zum Richter auf Lebenszeit) sowie der Beförderung von Richtern (Ernennung in höheren Richterstatus). Ferner berücksichtigt er die Praxis bei Abordnungen und Versetzungen.

\section{Kompetenz bei der Auswahl des richterlichen Nachwuchses (Einstellung)}

In 9 (von 16) Landesjustizverwaltungen hat das Ministerium die alleinige Kompetenz (Auswahl, Zeichnung der Urkunde). In Berlin, Bremen und Hamburg (Vorauswahl, Vorschlagsrecht) sowie in Sachsen-Anhalt (Beteiligung am Einstellungsgespräch im Ministerium) ist eine teilweise Beteiligung des OLG gegeben. In Niedersachsen hat eine Auswahlkommission (je eine Person von den Gerichten des Bezirks, der Staatsanwaltschaft und dem Ministerium) die Entscheidungsbefugnis (nur noch formale Zeichnung der Urkunde im Ministerium). In Brandenburg und Nordrhein-Westfalen (NRW) hat das OLG die alleinige Kompetenz (einschließlich der Zeichnung der Ernennungsurkunde). 
2. Kompetenz bei der Entlassung von Proberichtern und der Ernennung zum Richter auf Lebenszeit

Nur in NRW hat das OLG die alleinige Befugnis (einschließlich der Zeichnung der Urkunde). In allen sonstigen Ländern entscheidet das Ministerium (aber im Zusammenwirken mit dem OLG und auf Vorschlag des OLG / Zeichnung der Urkunde durch das Ministerium).

\section{Kompetenz bei der Entlassung auf eigenes Verlangen und bei der Versetzung in} den Ruhestand von Richtern auf Lebenszeit

Siehe dazu wie oben unter 2., jedoch mit der Ausnahme, dass in Berlin das Kammergericht für die Versetzung in den Ruhestand allein zuständig ist.

\section{Kompetenz bei der Beförderung von Richtern}

Die abschließende Befugnis zur Besetzung von Beförderungsstellen kommt in allen Bundesländern ausschließlich dem Ministerium zu. Das OLG bereitet aber die vom Ministerium zu treffende Entscheidung vor (Ausschreibung der Stelle [soweit vorgesehen], Einholung von Beurteilungen, eventuelle Überbeurteilung, Unterbreitung eines Vorschlags gegenüber dem Ministerium). Die Zeichnung der Urkunde erfolgt durch den Minister, je nach Richterstatus auch durch den Regierungschef (in BadenWürttemberg z.B. auch bei der Vergabe von R 2- Ämtern).

\section{Kompetenz zur Erstbeurteilung, zur Überbeurteilung / Wer hat das »letzte Wort«?}

Die Befugnis zur Erstbeurteilung liegt in allen Ländern beim jeweiligen Dienstvorgesetzten. Bis auf Baden-Württemberg (dort nur bei einem Widerspruch des Beurteilten) und Hamburg (keine »Zweitbeurteilung «) haben alle OLGe die Kompetenz zur Überbeurteilung). Eine weitere Überbeurteilung durch das Ministerium ist nirgendwo vorgesehen.

6. Kompetenz zur Abordnung von Richtern auf Probe an Gerichte des Bezirks bzw. auch an die Staatsanwaltschaft (je nach Land)

Nur in Berlin, Brandenburg, Niedersachsen, NRW, Rheinland-Pfalz, Sachsen-Anhalt und Schleswig-Holstein hat das OLG die Befugnis zur Abordnung.

\section{Kompetenz zur Abordnung und Versetzung von Richtern auf Lebenszeit}

Keine Befugnisse beim OLG gibt es in Baden-Württemberg, Bremen, Hamburg, Rheinland-Pfalz, Saarland, Sachsen und Thüringen. Abordnungskompetenzen für das OLG im Rahmen seines Geschäftsbereichs gibt es in Bayern, Berlin, Brandenburg, Mecklenburg-Vorpommern, Niedersachsen, Sachsen-Anhalt und Schleswig-Holstein. In Bayern hat das OLG die Befugnis zur Versetzung der R 1- Richter seines Bezirks. In NRW hat das OLG die volle Kompetenz bei der Abordnung und Versetzung. 


\section{Folgerungen für eine stärkere Selbstverwaltung durch mehr Selbstverantwortung}

Die Vielfalt der bundesdeutschen Justizverwaltungspraxis im Personalbereich belegt, dass eine stärkere Delegation von (dienstrechtlichen und praktischen) Befugnissen auf die Gerichte möglich ist. Ein solcher Kompetenzwechsel ist leicht realisierbar. Einer Änderung der Verfassung bedarf es ebenso wenig wie der Installation einer neuen (Doppel-) Bürokratie in der Justiz. Erforderlich ist allerdings eine Änderung im Selbstverständnis und Aufgabenzuschnitt der Ministerien. Eine Kompetenzerweiterung unterhalb der ministeriellen Ebene (hier: der Oberlandesgerichte, evtl. mit weiteren Delegationsmöglichkeiten) wäre eine deutliche Stärkung der Justiz. Diese verfügt über die notwendige Kompetenz, um mehr Selbstverantwortung zu übernehmen. Im Einzelnen ist anzumerken:

\section{Einstellungsverfahren}

Die Praxis in Brandenburg, Niedersachsen und NRW stellt in besonderer Weise unter Beweis, dass sich das Delegationsmodell bewährt hat. Weil die Kriterien des Auswahlverfahrens (fachliche und soziale Kompetenz nach definierten Anforderungsmerkmalen) im Zusammenwirken von Ministerium, Gerichten und Richtervertretungen erstellt und allseitig verbindlich sind, ist der erzielte Konsens zur Transparenz der Auswahlkriterien und zum allgemein bekannten Verfahrensablauf ein Faktor, der die Justiz generell stärkt.

\section{Anstellung auf Lebenszeit}

Die Diskussion, ob der Anstellung auf Lebenszeit die eigentliche »politische « Bedeutung im Richterauswahlverfahren zukommt, geht an den Realitäten des Verwaltungsalltags vorbei.

Wer als Proberichter die ersten Jahre »überstanden« hat, gilt in der Regel als so qualifiziert, dass die Lebenszeitanstellung sich auf einen rein formalen Akt reduziert. Deshalb sollte die Befugnis für die Entscheidung auf die OLGe übertragen werden (Aufgabendelegation).

\section{Beförderung von Richtern}

Auch die Beförderung kann in weiten Bereichen auf die Gerichte übertragen werden (jedenfalls bis zu Ämtern der Stufe »R 2+Zulage«). Weil die durch Zeugnisse ausgewiesene Kompetenz das wichtigste Auswahlkriterium ist (bzw. sein sollte), besteht keine sachlich gebotene Notwendigkeit, die Beförderungsentscheidung auf der Ebene der Ministerialverwaltung zu belassen. Eine »geborene Zuständigkeit « ist nur im traditionellen Sinn gegeben. Es handelt sich bei der größten Zahl der Beförderungsentscheidungen durchweg nur um das operative »Alltagsgeschäft « einer Personalverwaltung. Konzentriert ein Ministerium sein Aufgabengebiet auf das Kerngeschäft strategischer Grundsatzentscheidungen, können hier Kompetenzen sinnvoll verlagert werden. Eher strategische Aspekte spielen eine Rolle in der Regel nur bei der Vergabe von Behördenleitungen der Präsidialgerichte. Hier fokussiert sich politische Macht. 
Diesem Faktum muss sich die Justiz stellen, wenn sie Teil der gesellschaftlichen Realität bleiben will. Hätten andere Gremien diese Personalentscheidungen zu treffen, wäre gleichwohl keine Garantie gegen Proporzgerangel und fehlende Transparenz gegeben. Der Beigeschmack, der auch dann entstünde, wäre nur ein anderer. Nach meinen Erfahrungen orientiert sich die Kandidatenauswahl für Spitzenämter bei jeweils unterstellter fachlicher Kompetenz wesentlich am Persönlichkeitsbild, das für das jeweilige Amt gewünscht wird. Nicht ohne Belang sind dabei auch der Stil und die Beachtung einer justizorientierten »political correctness « in der politischen Praxis.

\section{Beurteilungswesen}

Wegen des bei Beförderungen zu beachtenden Prinzips der Bestenauslese nach Art. 33 GG kommt den Kriterien des Beurteilungswesens eine maßgebliche Rolle zu. Wenn unter »Selbstverwaltung « nicht verstanden wird, dass die Richter sich untereinander die Beurteilungen ausstellen sollen, muss die Befugnis dazu beim richterlichen Dienstvorgesetzten bleiben. Ein Beurteilungswesen ist aber nur so gut, wie es valide und transparente Kriterien als Grundlage hat. Je konkreter z. B. Zeugnisformulare einzelne Beurteilungsmerkmale benennen, zu denen sich der Beurteilende äußern muss, desto größer ist die Transparenz. Wenn diese Kriterien im Zusammenwirken von der Justizverwaltung, den Gerichten und der Richtervertretung erarbeitet werden, stärkt das die Akzeptanz in der Richterschaft. Ein Beurteilungswesen, das sich daran orientiert, lässt erwarten, dass sachfremde Aspekte bei Beförderungsentscheidungen weniger durchschlagen.

\section{Stärkung der richterlichen Mitbestimmung}

Um einer tatsächlichen oder partiell auch nur empfundenen »Fremdverwaltung « entgegenzuwirken, ist die Partizipation der Richterschaft an Entscheidungen der Gerichts- und der Justizverwaltung zu stärken. Das könnte einen vielerorts verloren gegangenen Vertrauensbonus seitens einer deutlich sensibler gewordenen Richterschaft wieder entstehen lassen. In den Mitwirkungsmöglichkeiten, die die Richtergesetze den von der Richterschaft gewählten Gremien eröffnen, kommt die richterliche Selbstverwaltung und Selbstverantwortung besonders zum Tragen. Dieser Kompetenzbereich kann und sollte ausgebaut werden. Dadurch würde zugleich das richterliche Mitverantwortungsgefühl gestärkt, auf das auch Behördenleitungen angewiesen sind. Eine in der Praxis schon jetzt nicht zu unterschätzende Bedeutung haben die von der Richterschaft gewählten Präsidialräte. Ihre Arbeit ist Ausdruck einer bereits praktizierten Selbstverwaltung. Ebenso wie diese derzeit ein Korrektiv bei Ministerialentscheidungen sein können, wären sie es bei einer Kompetenzsteigerung der Gerichte. Nur in seltenen Fällen setzt sich ein Minister mit einer Kabinettsentscheidung über das Votum des Präsidialrats hinweg. Dabei bestätigt die Ausnahme die Regel. Meist wird ein Konsens gesucht. »Fehlentscheidungen« gibt es im Übrigen nicht allein in der Politik. Sie wären in Ausnahmefällen auch bei veränderten Personalkompetenzen denkbar. Auch die Richterräte vor Ort gewinnen in Personalangelegenheiten eine zunehmende Bedeutung, indem sie im Vorfeld späterer Besetzungsentscheidungen in der Weise be- 
teiligt werden, dass sie z.B. bei der Auswahl von Teilnehmerinnen und Teilnehmern an Fortbildungsveranstaltungen im Rahmen von Personalentwicklungsmaßnahmen mitwirken.

\section{Appell zur Enthierarchisierung der Personalverwaltung}

In der Enthierarchisierung der Personalverwaltung liegt ein großes Reformpotential für die deutsche Justiz. Dieses sollte genutzt werden, um Politik und Justiz wieder näher zueinander zu führen, vor allem aber, um das Selbstwertgefühl der Richterschaft in einer weitgehend selbstverantworteten Justiz zu stärken. Eine Bereitschaft auf politischer Ebene ist im vernünftigen Dialog »herstellbar«, wie die Praxis in einigen Bundesländern - jedenfalls partiell - zeigt. Der Blick in andere europäische Länder hilft nur marginal. Die dortige Justizorganisation erklärt sich aus den jeweiligen historischen, politischen und gesellschaftlichen Rahmenbedingungen. Diese sind mit der (heutigen) deutschen Justiz- und Verfassungswirklichkeit nicht vergleichbar. Fangen wir bei uns selbst an. Es gibt genug zu tun! 